ITEP-PH-5/97, CPPM/97-3, INP-MSU 97-18/469

25 July 1997

\title{
On the search for muonic photons in neutrino experiments
}

\author{
V.A. Ilyin ${ }^{1)} * ，$ L.B. Okun ${ }^{2) \dagger} ，$ A.N. Rozanov $\left.{ }^{3}\right|^{\dagger}$ \\ * Institute of Nuclear Physics, Moscow State University, Moscow 119899, Russia \\ ${ }^{\dagger}$ Institute of Theoretical and Experimental Physics, Moscow 117218, Russia \\ ‡ CPPM, IN2P3-CNRS, Marseille, France
}

\begin{abstract}
Conserved muonic number may turn out to be a conserved muonic charge, coupled to muonic photons, $\gamma_{\mu}$. Muons and muonic neutrinos would emit $\gamma_{\mu}$ 's, which might be discovered by analysing the data from the past and future high energy neutrino experiments (like CHARM II, CCFR, CHORUS, NOMAD, etc.). There are two sources of $\gamma_{\mu}$ 's in these experiments: 1) internal bremsstrahlung in pion and kaon decays into $\mu$ and $\nu_{\mu}$, which provide neutrino beams; 2) external bremsstrahlung of muons in the shielding of the neutrino beam. In both cases the $\gamma_{\mu}$ 's would pass freely through the shielding and produce narrow muonic pairs in the neutrino detectors. These pairs could be distinguished from the trident events $\left(\nu_{\mu}+Z \rightarrow \nu_{\mu}+\mu^{+}+\mu^{-}+Z\right)$ by their kinematical properties: small $p_{t}$ of the muon pair, small invariant mass, etc. All the above processes are quantitatively analysed in this paper.
\end{abstract}

\section{Introduction}

Speculations on the existence of massless vector particles (extra photons) coupled to baryonic number [1] and leptonic numbers [2]-[何 have been under discussion for several decades. In particular, three types of such photons - electronic, $\gamma_{e}$, muonic, $\gamma_{\mu}$, and tauonic, $\gamma_{\tau}$, - have been considered recently in ref. [3, 7] where the search for muonic photons at high energy neutrino experiments was discussed. In the present paper we undertake quantitative analysis of such a search by considering both the emission and the detection of $\gamma_{\mu}$ 's.

As it is well known, the beams of muonic neutrinos in high energy neutrino experiments are formed in a long decay tunnel, where pions and kaons decay :

$$
\begin{aligned}
& \pi^{+} \rightarrow \mu^{+}+\nu_{\mu}, \quad \pi^{-} \rightarrow \mu^{-}+\bar{\nu}_{\mu} \\
& \mathrm{K}^{+} \rightarrow \mu^{+}+\nu_{\mu}, \quad \mathrm{K}^{-} \rightarrow \mu^{-}+\bar{\nu}_{\mu}
\end{aligned}
$$

( $\mathrm{K}_{\mu 3}$ decays are less important). The decay tunnel is followed by a thick shielding of iron, earth and concrete, which absorbs the beam of muons and all other secondary particles (hadrons and photons). For instance, in the neutrino beam of the CERN SPS the 185 meters of iron were followed by $173 \mathrm{~m}$ of earth, and again by $20 \mathrm{~m}$ of iron and $15 \mathrm{~m}$ of concrete [8]. The beam of muonic neutrinos passes through the shielding and produces reactions in the neutrino detector: mainly inelastic scattering on nuclei due to charged and neutral current interactions.

The idea of the search for muonic photons in neutrino experiments is based on the fact that the coupling constant of the muonic photons $\alpha_{\mu}$ is small compared to $\alpha=1 / 137$ :

$$
\alpha_{\mu} / \alpha<10^{-5} .
$$

This limit follows [2, 7] from the precision measurements of the $(g-2)_{\mu}$ - anomalous magnetic moment of the muon [9]. Because of smallness of $\alpha_{\mu}$, muonic photons would easily penetrate the shielding in the neutrino experiments and produce narrow muon pairs in the detector due to the reaction of the muon pair production by $\gamma_{\mu}$ in the Coulomb field of a nucleus with charge $Z$ :

$$
\gamma_{\mu}+Z \rightarrow \mu^{+}+\mu^{-}+Z .
$$

On the other hand, the rates of emission of muonic photons and of production of muon pairs by them would be also small, which does not promise an easy hunt.

The main background for the process (4) are the so-called tridents:

$$
\nu_{\mu}+Z \rightarrow \nu_{\mu}+\mu^{+}+\mu^{-}+Z \text {. }
$$

\footnotetext{
1) ilyin@theory.npi.msu.su

2) okun@vxitep.itep.ru

3) rozanov@cppm.in2p3.fr
} 
As we will show, the dimuon invariant mass distribution for tridents is broader, and these dimuons have much larger $p_{t}$-imbalance than in process (円), which may help to cope with the trident background.

To get accurate numerical results for the processes discussed in this paper we used the package CompHEP [10] to obtain analytical expressions for squared Feynman diagrams. For these purposes a new Lagrangian have been implemented to include new particle, muonic photon, and its interaction with muon and muonic neutrino. Then, for each process under the calculation, the FORTRAN code was generated for squared matrix element using the corresponding CompHEP option, as well as the phase space factor with proper regularization of kinematical singularities (see [11] for the description of regularization). The nuclear form-factors were implemented in this code as additional factors multiplying the matrix elements of reactions (4) and (5). The convolution with the spectra of muonic photons, muons or neutrinos was realized using the so called 'structure function' option of the CompHEP package. The phase space integration was performed with Monte-Carlo integrator BASES, while events were generated by the program SPRING, both programs are combined in the package BASES/SPRING [12]. The statistical error in our MC calculations was less than $1 \%$. The results of CompHEP calculations are compared with the results of special CERN Monte-Carlo program in section 6 of this paper.

In this paper we present the theoretical basis for the search for muonic photons in a generic high energy neutrino experiment. The actual analysis of experimental data is possible only for collaborations, which run the experiments.

\section{Emission of $\gamma_{\mu}$ 's in pion and kaon decays.}

The calculation of the internal bremsstrahlung in process (1) is straightforward, but the resulting spectrum has a simple analytical form only if one neglects the kinetic energy of the muon compared to $\left(m_{\pi}-m_{\mu}\right)$. Then for a pion decaying at rest we get:

$$
\begin{aligned}
d B\left(\pi \rightarrow \mu+\nu_{\mu}+\gamma_{\mu}\right) & \equiv \frac{d \Gamma\left(\pi \rightarrow \mu+\nu_{\mu}+\gamma_{\mu}\right)}{\Gamma(\pi \rightarrow \mu \nu)}= \\
& =\frac{2 \alpha_{\mu}}{\pi} \cdot \frac{d x}{x} \cdot\left[\left(1-x+\frac{1}{2} x^{2}\right) \cdot \ln \frac{2(1-x)}{m_{\nu_{\mu}} /\left(m_{\pi}-m_{\mu}\right)}-1+x\right],
\end{aligned}
$$

where $x=\omega / \omega_{\max }, \omega$ is the energy of the muonic photon and $\omega_{\max }=\left(m_{\pi}^{2}-m_{\mu}^{2}\right) / 2 m_{\pi} \approx m_{\pi}-m_{\mu}$. Expression (6) is infrared divergent, but this divergence has no adverse physical implications, because muonic photons of vanishing energy cannot produce muonic pairs. Another important feature of the expression (6) is its logarithmic dependence on the mass of the muonic neutrino. This logarithm is due to configurations in which the photon and neutrino momenta are collinear. Thus, if one is lucky, one can not only discover the muonic photon, but to measure the mass of the muonic neutrino, even if it is extremely tiny and otherwise inaccessible. Both above

\begin{tabular}{|c|l|c|c|c|}
\hline Decay mode & $x=\omega / \omega_{\max }$ & $m_{\nu_{\mu}}=100 \mathrm{KeV}$ & $m_{\nu_{\mu}}=1 \mathrm{eV}$ & $m_{\nu_{\mu}}=10^{-10} \mathrm{eV}$ \\
\hline \hline \multirow{5}{*}{$\pi \rightarrow \mu \nu_{\mu} \gamma_{\mu}$} & 0.9 & 0.367 & 1.37 & 3.39 \\
& 0.7 & 0.599 & 2.00 & 4.80 \\
& 0.5 & 1.01 & 3.25 & 7.74 \\
& 0.3 & 2.08 & 6.54 & 15.5 \\
& 0.2 & 3.50 & 10.9 & 25.6 \\
& 0.1 & 7.86 & 24.1 & 56.6 \\
\hline & 0.9 & 0.083 & 0.211 & 0.465 \\
& 0.7 & 0.127 & 0.304 & 0.657 \\
& 0.5 & 0.210 & 0.494 & 1.06 \\
& 0.4 & 0.289 & 0.675 & 1.45 \\
& 0.3 & 0.426 & 0.991 & 2.12 \\
& 0.2 & 0.712 & 1.64 & 3.51 \\
& 0.1 & 1.59 & 3.65 & 7.76 \\
& 0.07 & 2.35 & 5.37 & 11.4 \\
& 0.05 & 3.36 & 7.68 & 16.3 \\
& 0.04 & 4.25 & 9.70 & 20.6 \\
\hline
\end{tabular}

Table 1: Internal bremsstrahlung: differential branching $d B / d \omega$ (in units of $10^{-5} \mathrm{GeV}^{-1}$, assuming $\alpha_{\mu} / \alpha=10^{-5}$ ) as a function of the muonic photon energy, $\omega$, for three different values of the muon neutrino mass, $m_{\nu_{\mu}}$, in the rest frame of decaying meson. The maximum photon energy is $\omega_{\max }=\left(m_{\pi / \mathrm{K}}^{2}-m_{\mu}^{2}\right) / 2 m_{\pi / \mathrm{K}}$. 

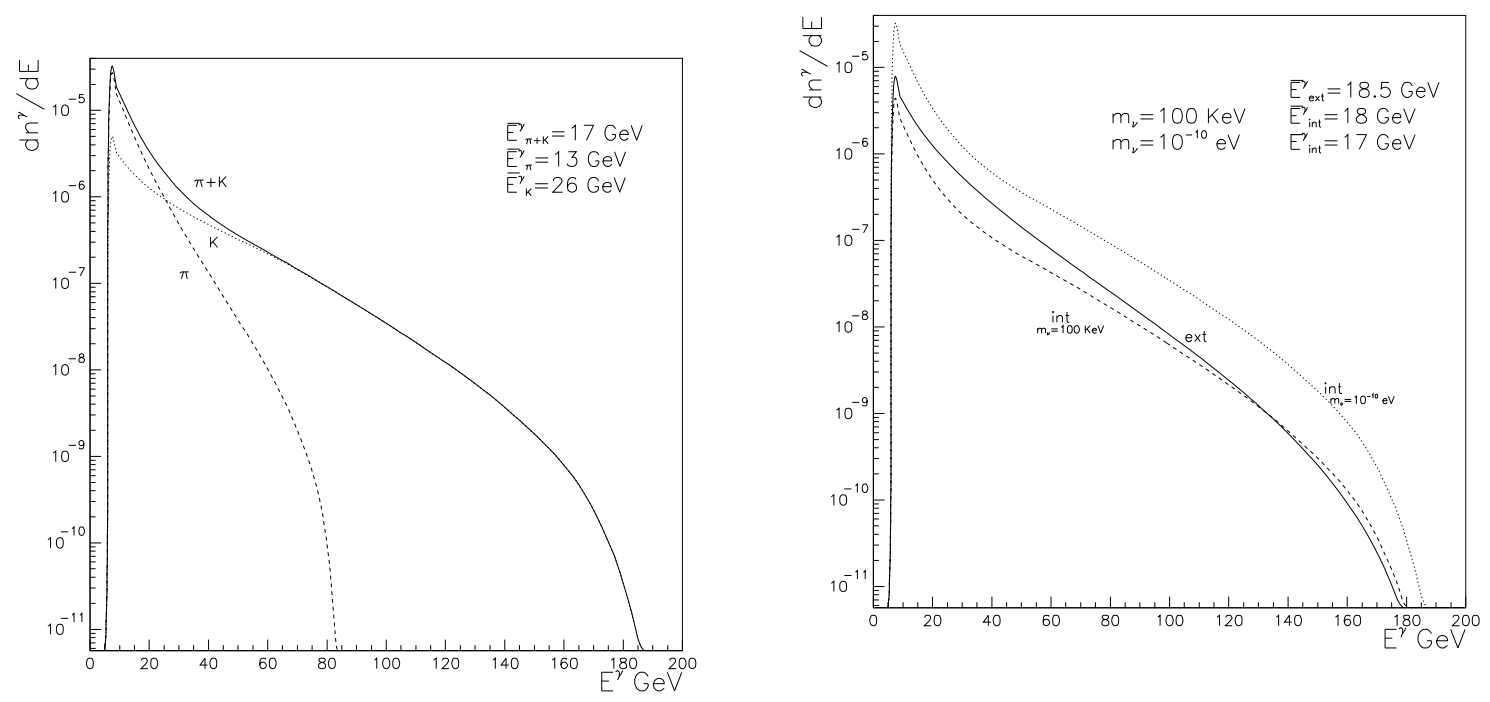

Figure 2: Comparison of the spectrum of muonic phoFigure 1: Spectra of muonic photons from internal tons from internal bremsstrahlung in the CERN 450 bremsstrahlung in the CERN $450 \mathrm{GeV}$ neutrino WBB (Wide Band Beam). The muon neutrino mass is assumed to be $m_{\nu}=10^{-10} \mathrm{eV}$.

GeV WBB neutrino beam for two masses of muon neutrino (dotted line $m_{\nu}=10^{-10} \mathrm{eV}$ and dashed line $m_{\nu}=100 \mathrm{KeV}$ ). The spectrum of muonic photons from external bremsstrahlung is shown by a solid line.

features (infrared and collinear) are characteristic for the spectrum of internal bremsstrahlung in a general case, when the kinetic energy of muon is taken into account. To obtain accurate numerical results for the differential branching, in particular in the case of K decay, we evaluated analytically the squared matrix element with the help of the package CompHEP [10]. Then, the integration over squared invariant mass of neutrino and $\gamma_{\mu}$ was done analytically using the computer algebra system REDUCE [13]. The obtained expression was evaluated numerically with high accuracy by the corresponding REDUCE option. The results of these accurate numerical calculations, differ from (6) by a few percent in the case of pion. For the pion and kaon decays they are presented in table 1 for a set of neutrino masses, assuming $\alpha_{\mu}=10^{-5} \alpha$.

A muonic photon with energy $\omega$ in the rest frame of a meson (pion or kaon) and angle $\theta$ (in the same frame) with respect to the direction of the meson beam has in the laboratory frame energy $E_{\gamma_{\mu}}$, where

$$
E_{\gamma_{\mu}}=\omega \gamma(1+v \cos \theta)
$$

Here $v$ is the velocity of the meson and $\gamma=1 / \sqrt{1-v^{2}}$. The spectrum of muonic photons is derived by integrating over $d \cos \theta$ and convoluting with the spectra of decayed pions and kaons. Photons are emitted within a cone with an angle $\theta^{\prime} \sim 1 / \gamma$. Thus, one should take into account the angular acceptance of the detector determined by the geometry of experiment (the length of the decay tunnel and the position and cross dimension of the detector). In this paper we use as an example the CHARM II neutrino detector at the $450 \mathrm{GeV}$ CERN SPS neutrino beam. This detector is located at $882 \mathrm{~m}$ from the $\pi / \mathrm{K}$ production target with the length of the decay tunnel of $414 \mathrm{~m}$. The formation of the $\gamma_{\mu}$ spectrum is discussed in [14] in details in the framework of a simplified approach to the simulation of the detector geometry and meson beam. As an example, we used the meson spectra from ref. [8] with the effective $\mathrm{K} / \pi$-ratio equal 0.24 . 4

The resulting spectra of muonic photons, produced by the internal bremsstrahlung, are presented in fig. 11.

An important feature of fig. 11 is that hard muonic photons are mostly produced in the kaon decays. Of course, it depends on geometrical parameters of the experiment (see [14]). However this feature could be taken into account in future experiments to increase possible yield of muonic photons produced by the internal bremsstrahlung.

In order to see the dependence of rates and spectra on the mass of the muon neutrino we present in fig. 2 the spectra of muonic photons for two values of the neutrino mass: $m_{\nu_{\mu}}=100 \mathrm{KeV}$ - close to the experimental limit

4) We made the calculations only for the neutrino component of the neutrino beam (focused positive mesons). Similar calculations should be done for the antineutrino admixture due to wrong sign mesons and for antineutrino beam (negative mesons focused), where we expect similar results. Also we note that in this semi-analytical approach we neglect angular and radial distributions of mesons and assume the uniform distribution of the meson decay point along the decay tube. The exact account for these effects and their correlations is possible by pure Monte-Carlo simulations and is presented in section 6 . 
[9], and another assumed to be very small, $m_{\nu_{\mu}}=10^{-10} \mathrm{eV}$. The yield of muonic photons is about seven times higher for the second choice of $m_{\nu_{\mu}}$. This allows one to hope that the mass of muonic neutrino could be measured in the experiments discussed, if muonic photon is discovered.

Emission of $\gamma_{\mu}$ 's by muons in the shielding.

The effective material for the muon bremsstrahlung in the shielding is iron:

$$
\mu+\mathrm{Fe} \rightarrow \mu+\gamma_{\mu}+\mathrm{Fe} .
$$

The cross-section of the bremsstrahlung for the electron has been calculated by Bethe and Heitler [15, 16]; its derivation may be found in many text-books, e.g. [17, 18]. In the case of muon there is an obvious substitution $m_{e} \rightarrow m_{\mu}$. Moreover, the nuclear form-factor must be accounted for. This has been done by a group at the Moscow Engineering Physics Institute (MEPhI) [19]-[22], see also [23]-[25]. In order to apply their expressions to the process ( 8 ) we have to multiply them by $\alpha_{\mu} / \alpha$. In the ultra-relativistic limit, when $E_{1} \gg m_{\mu}$ and $E_{2} \gg m_{\mu}$, where $E_{1}\left(E_{2}\right)$ is the energy of the initial (final) muon, we get:

$$
d \sigma=\alpha_{\mu} \frac{4}{3}\left(\frac{2 Z \alpha}{m_{\mu}}\right)^{2} \frac{d E_{\gamma_{\mu}}}{E_{\gamma_{\mu}}}\left[1-\frac{E_{\gamma_{\mu}}}{E_{1}}+\frac{3}{4}\left(\frac{E_{\gamma_{\mu}}}{E_{1}}\right)^{2}\right] \cdot\left[\ln \frac{2 E_{1}\left(E_{1}-E_{\gamma_{\mu}}\right)}{m_{\mu} E_{\gamma_{\mu}}}-\frac{1}{2}-1.5\right] .
$$

Here $E_{\gamma_{\mu}}$ is muonic photon energy, $Z$ is the charge of a nucleus of iron $(Z=26)$. The term 1.5 appears due to the nuclear form-factor, which was assumed in the form 5 :

$$
F\left(q^{2}\right)=\exp \left(-q^{2} a^{2} / 6\right), \quad a=\left(0.58+0.82 A^{1 / 3}\right) \mathrm{fm} .
$$

Another parametrization of the nuclear form-factor correction is suggested in ref. [22], according to which, instead of 1.5 in the second bracket of eq. (9), one has to substitute

$$
\Delta_{n}^{e l}(\delta)=\ln \frac{D_{n}}{1+\delta\left(D_{n} \sqrt{e}-2\right) / m_{\mu}},
$$

where $D_{n}=1.54 A^{0.27}, A$ - mass number of the nucleus $\left(A=56\right.$ for iron), $\delta=m_{\mu}^{2} E_{\gamma_{\mu}} / 2 E_{1}\left(E_{1}-E_{\gamma_{\mu}}\right)-$ minimal momentum transfer, and $e=2.718$. The final numerical results of eqs. (9) and (11) are close to each other. In this paper we do not take into account the atomic screening and inelastic form-factors, both nuclear and atomic (see ref. [22]).

The total cross-section of the process $(8)$ convoluted with the spectrum of muons emitted in pion and kaon decays equals to $1760 \mathrm{pb}$ for $\alpha_{\mu}=10^{-5} \alpha$. This result was obtained with the help of programs CompHEP and BASES. To calculate the spectrum of muonic photons emitted by muons in the iron shielding we used the formula (9) convoluted with the spectrum of muons. The spectra of muonic photons, produced by the external bremsstrahlung in the iron shielding are presented in fig. 3. The internal and external spectra are compared in fig. 2. First of all, one can see that the yield of muonic photons produced by the internal bremsstrahlung is higher for $m_{\nu_{\mu}}=10^{-10} \mathrm{eV}$ than the yield by the external bremsstrahlung, supporting prospects to measure muonic neutrino mass if $\gamma_{\mu}$ is discovered. The internal bremsstrahlung spectra are harder than the external ones. Thus increasing the energy of mesons (especially of kaons) should help in measuring neutrino mass. Moreover, the ratio of pion and kaon components is drastically different for muons (and hence for their bremsstrahlung in the shielding) from that for the mesons themselves. Indeed, while initial meson spectrum in the CERN beam has $80.4 \%$ decayed pions, the muon flux at the end of the decay tunnel has $96.5 \%$ muons emitted in the pion decays. The relative enhancement of pion component is caused by a strong suppression of the kaon component, because the muons and muonic gammas from K-decays have wider angular distribution and are partly missing the acceptance of the neutrino detector (see [14] for the corresponding formulas). However the contribution of energetic muons from kaon decays is enhanced by rather high energy cut, $7 \mathrm{GeV}$, on muonic photons and the increase of the bremsstrahlung cross-section with energy. Finally, $82.9 \%$ of muonic photons and $80.3 \%$ of muon pairs originate from pions (this effect is discussed in more details in ref. [14]).

In the external bremsstrahlung calculations we have neglected angular distribution of muonic photons in the reaction (8). Indeed, muons, moving at angles larger than the angular size of the detector could, nevertheless, produce muonic photons crossing the detector. Moreover, muons, moving in the directions crossing the detector, could produce muonic photons going outside it. We assume that these two effects more or less compensate each other. The angular distribution of muonic photons produced in the external bremsstrahlung has a forward peak with characteristic polar angle $\theta \sim m_{\mu} / \bar{E}_{1}$, where $\bar{E}_{1}$ is the muon average energy. For $\bar{E}_{1}=31 \mathrm{GeV}$, more than $80 \%$ of photons lie inside this $\theta$-cone (about $0.2^{\circ}$ ). Thus, we assume that the effect under discussion should give a correction factor not larger than 2 in our final results.

5) An extensive compilation of nuclear elastic form-factors can be found in ref. [26]. 

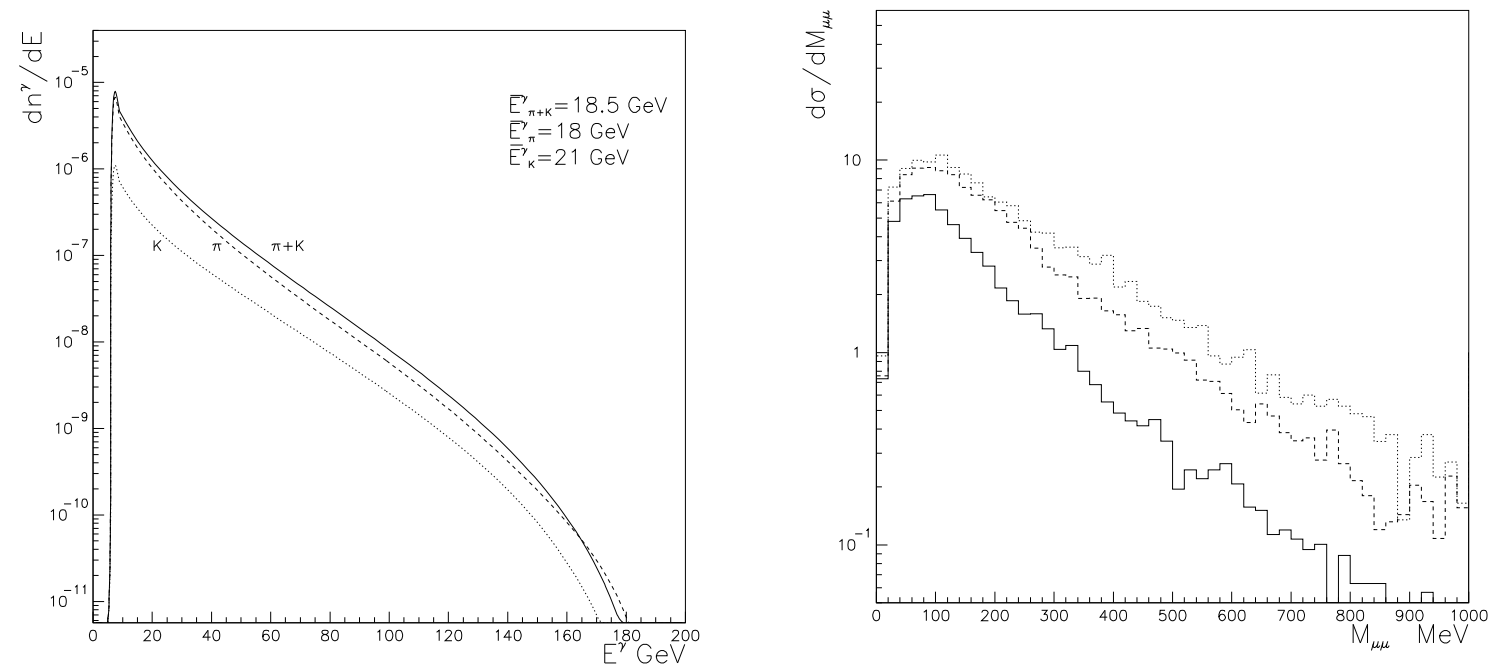

Figure 3: Spectra of muonic photons from external bremsstrahlung in the CERN $450 \mathrm{GeV}$ WBB neutrino beam. The contribution from $\pi$-decay is shown as dashed line, from K-decays by dotted line.

Figure 4: Invariant mass distributions (in units of $\mathrm{pb} / 20$ $\mathrm{MeV}$ ) of muon pair from the conversion in the neutrino detector of muon gammas with fixed energies of $20 \mathrm{GeV}$ (solid), $50 \mathrm{GeV}$ (dashed) and $100 \mathrm{GeV}$ (dotted line).

\section{Photoproduction of muon pairs by $\gamma_{\mu}$ 's.}

The photoproduction of muons by ordinary photons has been analysed in a number of papers. The corresponding cross-section is connected with the bremsstrahlung cross-section by crossing (see refs. [17, 18]). One can use the differential cross-section suggested in ref. [27], substituting $\alpha_{\mu}$ instead of $\alpha$, but keeping the factor $Z \alpha$

$$
\begin{aligned}
d \sigma\left(\gamma_{\mu}+Z \rightarrow \mu^{+}+\mu^{-}+Z\right) & =\alpha_{\mu}\left(\frac{2 Z \alpha}{m_{\mu}}\right)^{2}\left(1-\frac{4 x_{+} x_{-}}{3}\right) d x_{+} \times \\
& \times\left[\ln \left(\frac{2}{3} \frac{m_{\mu}}{m_{e}} \frac{A_{0}}{Z^{2 / 3}}\right)-\ln \left(1+\frac{A_{0}}{Z^{1 / 3}} \frac{\delta \sqrt{e}}{m_{e}}\right)\right] .
\end{aligned}
$$

Here $\alpha=1 / 137, Z$ is atomic number of the nucleus $(Z=10$ for the 'average nucleus' in the CHARM II detector), $\delta=m_{\mu}^{2} / 2 E_{\gamma_{\mu}} x_{+} x_{-}-$minimal momentum transfer, $E_{\gamma_{\mu}}-$ the energy of the muonic photon, $x_{ \pm}=$ $E_{ \pm} / E_{\gamma_{\mu}}$, where $E_{ \pm}$- energy of $\mu^{ \pm}, A_{0} \simeq 190-$ a constant, which determines the value of the radiation logarithm.

With $E_{\gamma_{\mu}} \sim 20 \mathrm{GeV}$ and $0.1<x_{ \pm}<0.9$, the atomic screening may be neglected, so that the logarithmic factor simplifies:

$$
\ln \frac{2}{3} \frac{m_{\mu}}{\delta Z^{1 / 3} \sqrt{e}}=\ln \frac{4}{3} \frac{E_{\gamma_{\mu}} x_{+} x_{-}}{m_{\mu} Z^{1 / 3}}-\frac{1}{2} .
$$

Another, more recent parametrisation of the same cross section[6] is:

$$
d \sigma=\alpha_{\mu}\left(\frac{2 Z \alpha}{m_{\mu}}\right)^{2}\left(1-\frac{4 x_{+} x_{-}}{3}\right)\left[\ln \frac{\frac{B}{Z^{1 / 3}} \frac{m_{\mu}}{m_{e}}}{1+\frac{B}{Z^{1 / 3}} \frac{\delta}{m_{e}} \sqrt{e}}-\ln \frac{D_{n}}{1+\frac{\delta}{m_{\mu}}\left(D_{n} \sqrt{e}-2\right)}\right] d x_{+},
$$

where $B=183, D_{n}=1.54 \cdot A^{0.27}$ and $A$ is a number of nucleons in a nucleus $(A=20$ for the 'average nucleus' of the CHARM II detector). Note that for not too small values of the $\delta$ parameter $\left(\delta / m_{e} \gg Z^{1 / 3} / B \sqrt{e}\right)$ the square brackets in eq. (14) reminds the last factor of eq. (9) with substitution of the term $(-1.5)$ by eq. (11):

$$
\{\} \rightarrow\left[\ln \left(\frac{m_{\mu}}{\delta}\right)-\frac{1}{2}-\ln \frac{D_{n}}{1+\delta\left(D_{n} \sqrt{e}-2\right) / m_{\mu}}\right] \text {. }
$$

6) S.R.Kelner, R.P.Kokoulin, private communication 

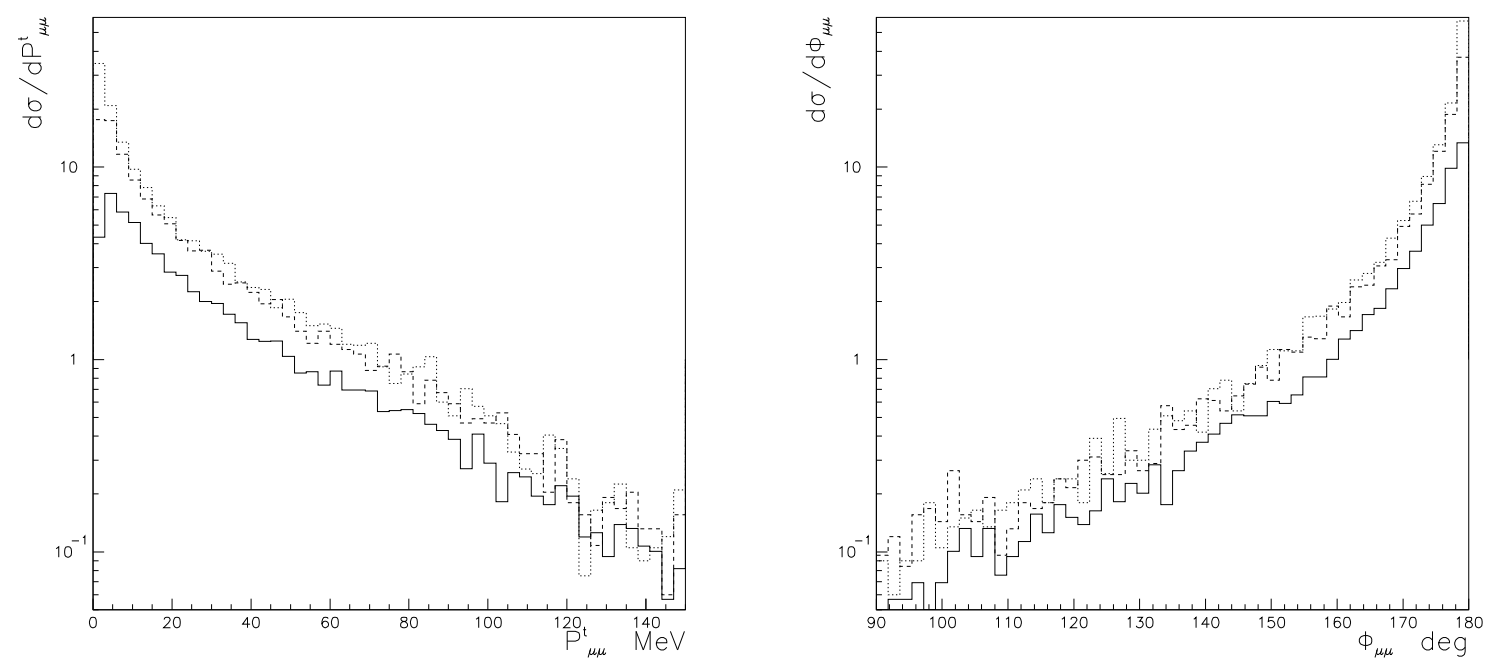

Figure 5: Transverse momentum distributions (in units of $\mathrm{pb} / 3 \mathrm{MeV}$ ) of muon pair from the conversion in the neutrino detector of muon gammas with fixed energies of $20 \mathrm{GeV}$ (solid), $50 \mathrm{GeV}$ (dashed) and $100 \mathrm{GeV}$ (dotted line).
Figure 6: Azimuthal angle distributions (in units of $\mathrm{pb} / 1.8^{\circ}$ ) between two muons from the conversion in the neutrino detector of muon gammas with fixed energies of $20 \mathrm{GeV}$ (solid), $50 \mathrm{GeV}$ (dashed) and $100 \mathrm{GeV}$ (dotted line).

Note also that if we neglect both atomic screening and nuclear form-factor and integrate over $d x_{+}$, we reproduce an expression similar to the well known result [ref. 13, eq. 94.6]:

$$
\sigma=\alpha_{\mu} \frac{7}{9}\left(\frac{2 Z \alpha}{m_{\mu}}\right)^{2}\left(\ln \frac{2 E_{\gamma_{\mu}}}{m_{\mu}}-\frac{109}{42}\right) .
$$

Similarly to the case of the muon bremsstrahlung in the shielding the nuclear elastic form-factor can be accounted for effectively by introducing an additional term in brackets, -1.2 . This value can be obtained from the parametrisation (10) for average nuclei with $Z=10$, 'neon', in the CHARM II detector. Note that this term equals to -1.5 for $\mathrm{Fe}(Z=26)$, see $(9)$.

To get accurate numerical results for cross-section and to calculate distributions for dimuon photoproduction we used the programs CompHEP and BASES/SPRING . In particular, the total cross-section of dimuon photoproduction by an "average" muonic photon, for $\alpha_{\mu}=10^{-5} \alpha$, is $41.5 \mathrm{pb}$ in the case of convolution with the normalized spectrum of muonic photons produced by the external bremsstrahlung, and $35.6 \mathrm{pb}$ in the case of the internal bremsstrahlung (with $m_{\nu_{\mu}}=10^{-10} \mathrm{eV}$ ). Note that in the case of internal bremsstrahlung the shapes of all distributions depend only slightly on the neutrino mass. Of course this is caused by the similarity of muonic photon spectra for different neutrino masses (see fig. 2). This similarity can be easily understood as the main contribution comes from the logarithmic terms like that in eq. (6).

Returning to the formula (16) we note that its modified version, with additional term -1.2 effectively accounting for elastic nuclear form-factor, differs from the accurate calculation by CompHEP and BASES by less than $1 \%$ for $E_{\gamma_{\mu}}>50 \mathrm{GeV}$, and this difference does not exceed $10 \%$ for lower energies within the energy range $E_{\gamma_{\mu}}>7 \mathrm{GeV}$ discussed here.

Muons should have high enough energy to be detected; in our calculations we took the detector energy threshold for muons as $4 \mathrm{GeV}$. Keeping in mind this energy threshold we have applied energy cut $7 \mathrm{GeV}$ on the energies of the original muons and neutrinos and on the energy of muonic photons. However for the final muons, produced in the detector by muonic photons, we have applied cut $E_{\mu}>4 \mathrm{GeV}$. All values of the photoproduction cross-section given above were obtained with these cuts.

Taking into account the yields of muonic photons, normalized to one muon neutrino from two-body $\pi / \mathrm{K}$ mesons decays, calculated as discussed above (see [14]),

$$
\begin{array}{ll}
3.1 \cdot 10^{-7} & \text { ext. brems., } \\
1.5 \cdot 10^{-7} & \text { int. brems., } m_{\nu_{\mu}}=100 \mathrm{KeV}, \\
10.4 \cdot 10^{-7} & \text { int. brems., } m_{\nu_{\mu}}=10^{-10} \mathrm{eV},
\end{array}
$$

one can derive the the specific yields of muon pairs per unit neutrino flux: 

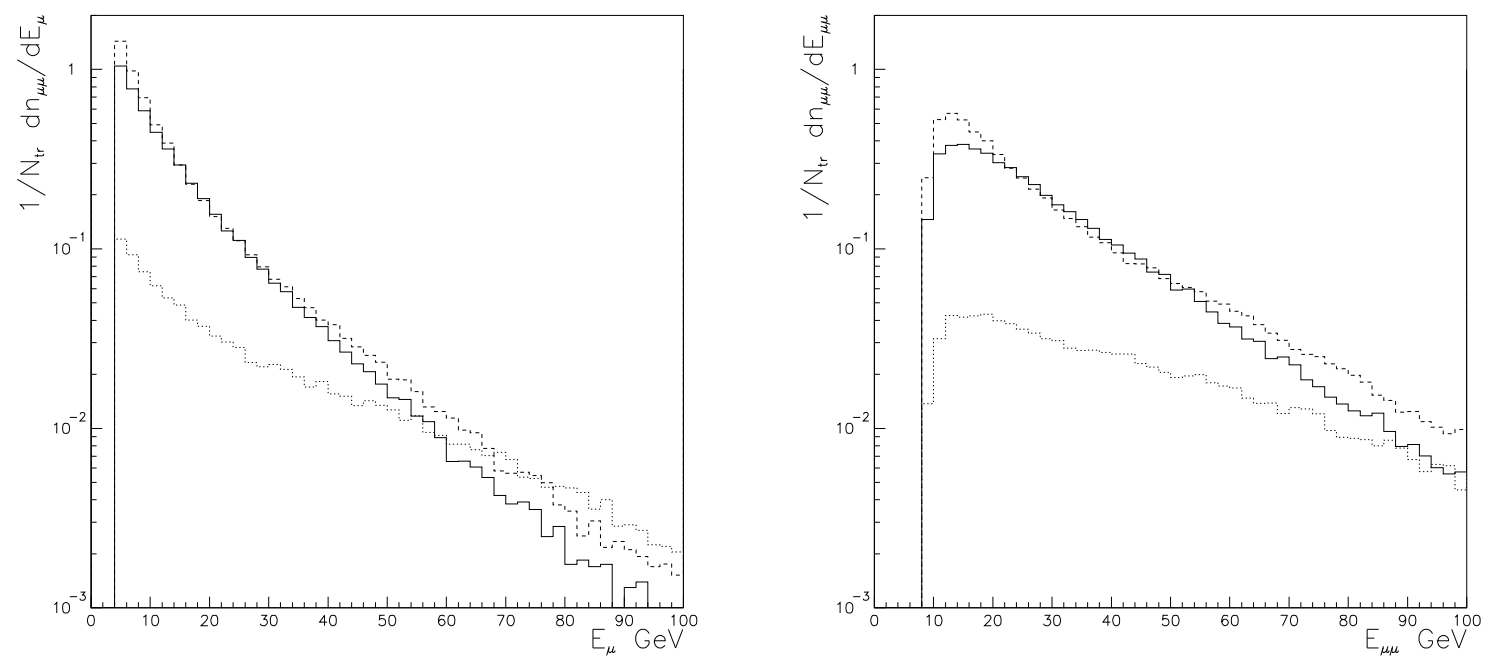

Figure 7: Energy spectra of muons from the conversion of muonic photons and from tridents in the neutrino detector at CERN neutrino beam. Solid line corresponds to external brems, dashed line to internal brems and dotted line to tridents.

Figure 8: Energy spectra of muon pairs from the conversion of muonic photons and from tridents in the neutrino detector at CERN neutrino beam. Solid line corresponds to external brems, dashed line to internal brems and dotted line to tridents.

$$
\begin{array}{ll}
2.1 \cdot 10^{-10} & \text { ext. brems. events } \mathrm{cm}^{2} / \text { neutrino, } \\
0.9 \cdot 10^{-10} & \text { int. brems. events } \mathrm{cm}^{2} / \text { neutrino, } m_{\nu_{\mu}}=100 \mathrm{KeV}, \\
5.9 \cdot 10^{-10} & \text { int. brems. events } \mathrm{cm}^{2} / \text { neutrino, } m_{\nu_{\mu}}=10^{-10} \mathrm{eV} .
\end{array}
$$

These specific yields of muon pairs are not exactly proportional to the specific yields of muonic photons because of the difference of the mean energies and effective cross-sections. To obtain these numerical results we took the number of average nuclei (with $Z=10$, 'neon') in the CHARM II detector equal to $0.16 \cdot 10^{32}$ [ 8 ], and $\alpha_{\mu}=10^{-5} \alpha$. Neutrino flux in CHARM II experiment is estimated [28] as $8.6 \cdot 10^{11} \nu / \mathrm{cm}^{2}$ integrated over the whole energy spectrum. So we would expect 177 dimuon pairs in CHARM II exposure from muonic photons produced by external bremsstrahlung. Then, in the case of internal bremsstrahlung we should observe about 74 events, if the mass of muonic neutrino is close to the experimental limit, $100 \mathrm{KeV}$, and more than 511 events, if the mass of muonic neutrino is very small, of order $10^{-10} \mathrm{eV}$. Invariant mass and transverse momentum of muon pairs, azimuthal angle between two muons distributions are shown on figs. 4, 5, 6 at some fixed energies of muonic photons: $20 \mathrm{GeV}$ (solid lines), $50 \mathrm{GeV}$ (dashed lines) and $100 \mathrm{GeV}$ (dotted lines).

The shapes of the transverse momentum and azimuthal angle distributions scales with energy, while the invariant mass of two muons slightly increases at high energy.

The energy spectra of muons and muon pairs from tridents and from conversion of muonic photons at CERN neutrino WBB are presented in figs. 7 and 8 .

In these and following figures we represent external brems by solid lines, internal brems with $m_{\nu_{\mu}}=10^{-10}$ $\mathrm{eV}$ by dashed lines and tridents by dotted lines. All distributions are normalized to one trident event. One can note that these spectra are harder in the case of the internal bremsstrahlung than in the external one.

One can see on fig. 9 that muons from $\gamma_{\mu}$ conversions are produced at small angles: more than $80 \%$ at angles less than $1^{\circ}$ with respect to the direction of generic muonic photon. We have found also that in both cases (external and internal ones) muons are produced with rather small $p_{t}: 90 \%$ of muons have transversal momentum less than $200 \mathrm{MeV}$ (fig. 10).

We also calculated distributions in different kinematical variables to find most effective cuts to localize the effect of muonic photons. In figs. 11, 12, 13, 14 we present the distributions in the invariant mass of dimuons, in the transversal momentum of a muon pair (the so-called $p_{t}$-imbalance), in the azimuth angle between muons (it is an angle between the transversal components of $\mu^{+}$and $\mu^{-}$momenta), and in the angle between total momenta of $\mu^{+}$and $\mu^{-}$.

\section{$5 \quad$ The trident background.}

As it has been mentioned in the introduction, the main background for the search of muonic pairs produced by muonic photons is presented by tridents, process $(5)$. The cross-section of trident production in a Coulomb field 

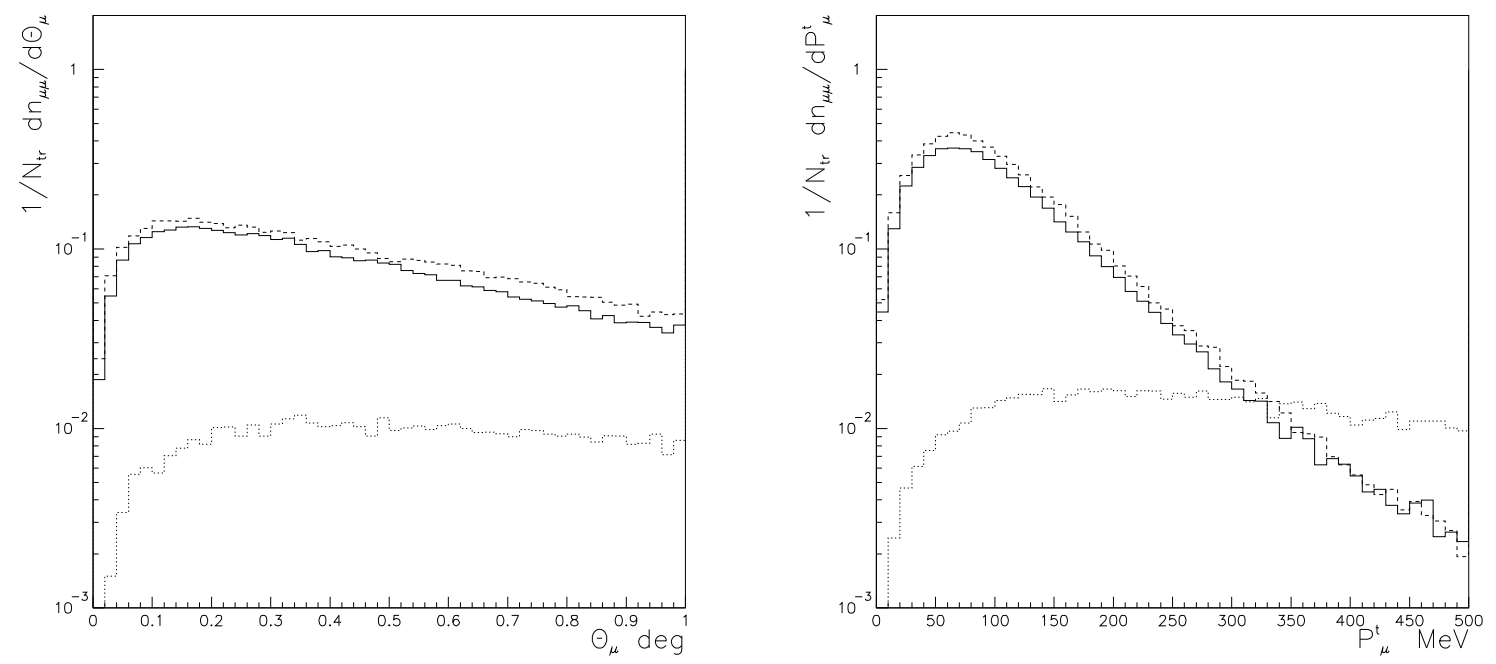

Figure 9: Angular distribution of muons from the conversion of muonic photons and from tridents in the neutrino detector at CERN neutrino beam. Solid line corresponds to external brems, dashed line to internal brems and dotted line to tridents.

Figure 10: Transverse momentum of muons from the conversion of muonic photons and from tridents in the neutrino detector at CERN neutrino beam. Solid line corresponds to external brems, dashed line to internal brems and dotted line to tridents.
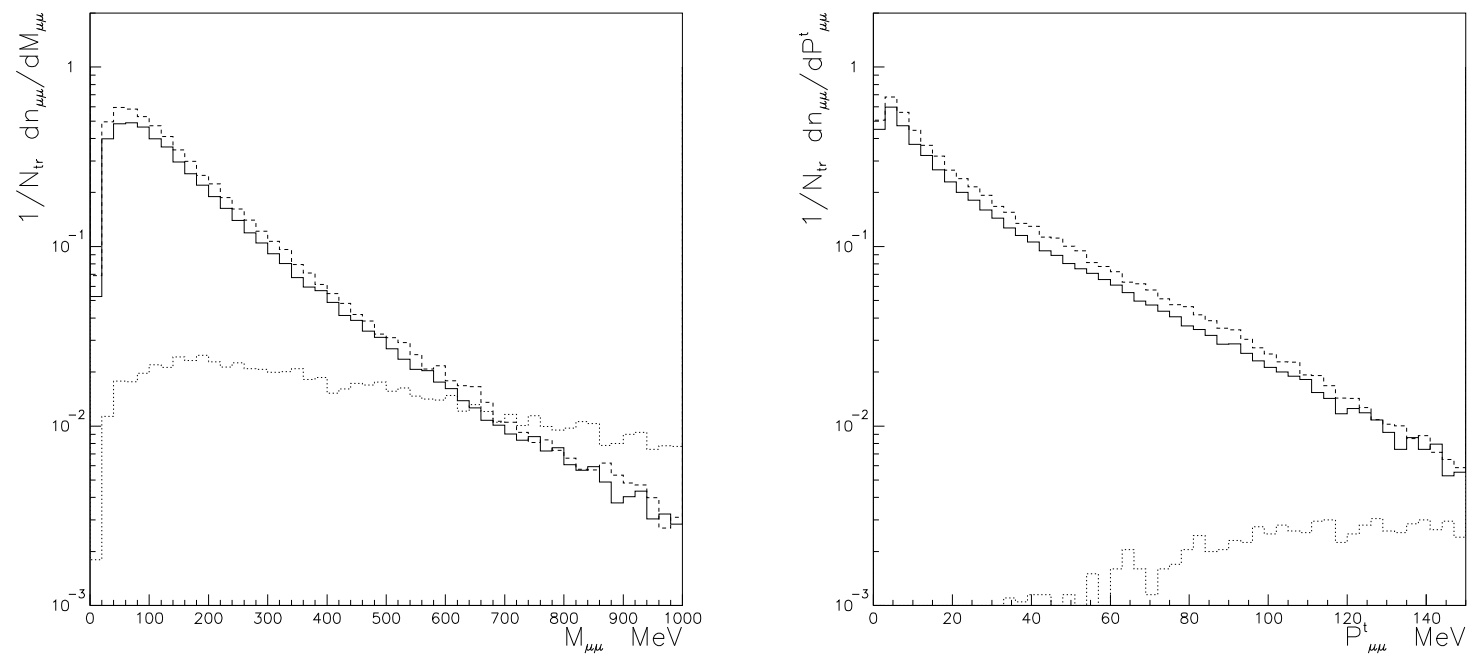

Figure 11: Invariant mass of muons from the conversion of muonic photons and from tridents in the neutrino detector at CERN neutrino beam. Solid line corresponds to external brems, dashed line to internal brems and dotted line to tridents.

Figure 12: Transverse momentum of muon pair from the conversion of muonic photons and from tridents in the neutrino detector at CERN neutrino beam. Solid line corresponds to external brems, dashed line to internal brems and dotted line to tridents. 


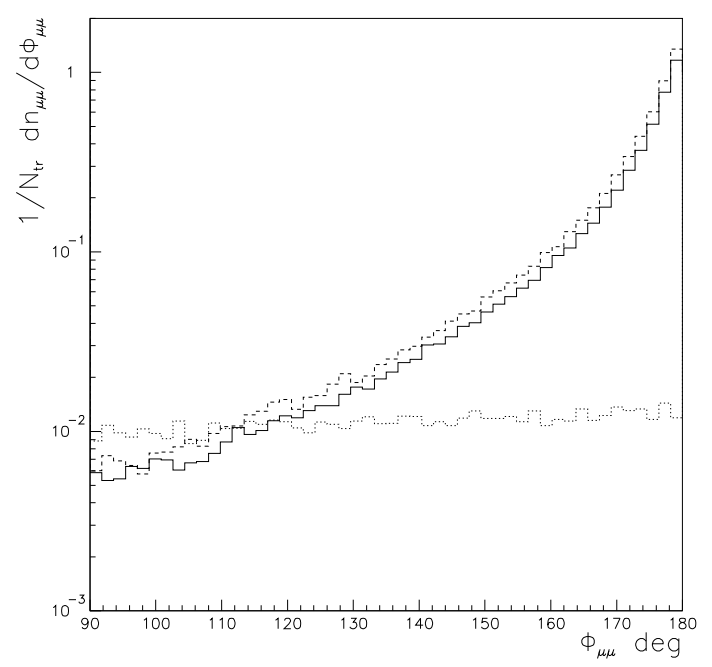

Figure 13: Azimuthal angle between muons from the conversion of muonic photons and from tridents in the neutrino detector at CERN neutrino beam. Solid line corresponds to external brems, dashed line to internal brems and dotted line to tridents.

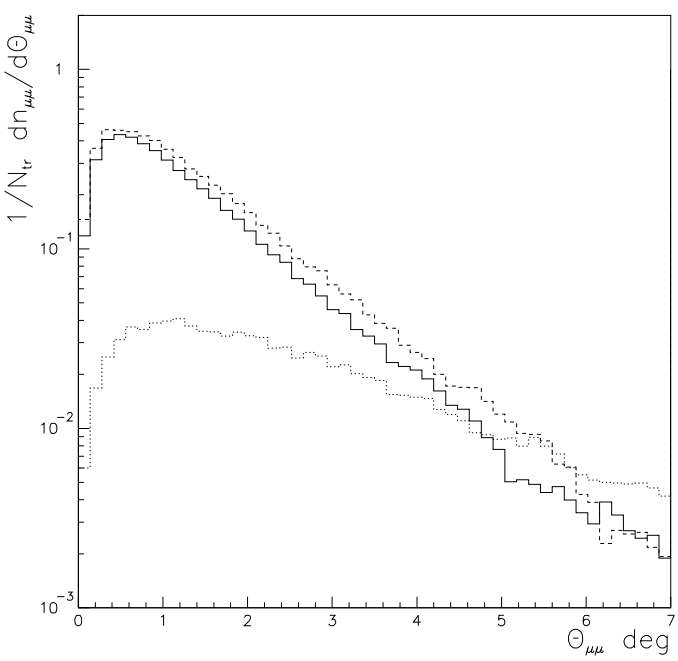

of a nucleus with charge $Z$, can be easily estimated using Weizsaecker-Williams approximation (see e.g. [29]):

$$
\sigma\left(\nu_{\mu}+Z \rightarrow \nu_{\mu}+\mu^{+}+\mu^{-}+Z\right)=\frac{2(Z \alpha)^{2} G^{2}}{9 \pi^{3}}\left(g_{L}^{2}+g_{R}^{2}\right) \cdot s_{\max } \cdot \ln \frac{s_{\max }}{4 m_{\mu}^{2}}
$$

Here $G=1.166 \cdot 10^{-5} \mathrm{GeV}^{-2}$ is the four-fermion coupling constant, $g_{L}=\frac{1}{2}+\sin ^{2} \theta_{W}, g_{R}=\sin ^{2} \theta_{W}$, $\sin ^{2} \theta_{W}=0.23, s_{\max }=2 E_{1} Q_{\max }, Q_{\max }=1 / a$, where $a$ is the radius of the nucleus, see eq. (10); $E_{1}$ is the energy of the initial neutrino. This approximate formula gives the value smaller by $\sim 10 \%$ then the accurate calculation using CompHEP and BASES within the discussed energy range. The cross-section convoluted with the normalized neutrino spectrum gives the value $\sigma=0.045 \cdot 10^{-4} \mathrm{pb}$. This numerical result has been obtained with the nuclear form-factor 10 and with energy cut $E_{\mu}>4 \mathrm{GeV}$ applied on muons. The number of trident events is estimated by using the neutrino flux and target mass given in Section 7 to be 50 events.

Energy spectra of muons, and muon pairs for tridents are presented by dotted lines in figs. 7 and 8 . In figs. 11, 12, 14, 13 we present distributions (also by dotted lines) in different variables for tridents - in invariant mass of the muon pair, in transversal momentum of the muon pair ( $p_{t}$-disbalance), in the angle between muon and antimuon, and in azimuth angle between muon and antimuon. One can see that trident events are distributed in these variables broader than muon pairs in dimuon photoproduction. The characteristic values of kinematical cuts on these variables are collected in table 2, together with the percentage of the background trident events which survive these cuts. It is obvious that the most promising is the cut in the transversal momentum of muon pair, $p_{t}$-disbalance. Only $2.12 \%$ of trident events have $p_{t}^{\mu \mu}<70 \mathrm{MeV}$. By applying this cut one can hope to get the suppression of trident background by a factor 50 while practically all muon pairs from photoproduction will be kept.

The cuts on invariant mass of muon pair and on azimuth angle between two muons could be also effective, each could suppress trident background by a factor 3-4. In addition, we found that distribution in transversal momentum of muons is much broader for tridents than for dimuon photoproduction, here the suppression factor 4 could be also obtained for tridents. Of course special analysis is necessary to find those combinations of these cuts which will give maximal suppression factor for the trident background - the correlations are important.

\section{Monte-Carlo simulation of the production and detection of the muonic photons.}

In order to take into account the details of the geometry and kinematics of the neutrino beam line, a special CERN Monte-Carlo program was used, which directly simulates the decay of $\pi / \mathrm{K}$ with muonic photon and the bremsstrahlung of muonic photons in the iron shielding of the neutrino beam. This program uses the Monte-Carlo $\pi /$ K-decays produced by GBEAM program [8] with realistic geometry, magnetic focusing in the horn and reflector 


\begin{tabular}{|l|c|c||c|}
\hline Observable & int.brems. & ext.brems. & tridents \\
\hline$M_{\mu \mu}(\mathrm{MeV})<$ & 500 & 500 & $39 \%$ \\
$p_{t}^{\mu \mu}(\mathrm{MeV})<$ & 72 & 73 & $2.12 \%$ \\
$p_{t}^{\mu}(\mathrm{MeV})<$ & 200 & 200 & $24.9 \%$ \\
$\varphi_{\mu \mu}(\mathrm{deg})>$ & 142 & 142 & $25.6 \%$ \\
$\vartheta_{\mu \mu}(\mathrm{deg})<$ & 2.8 & 2.7 & $64.2 \%$ \\
$E_{\mu}(\mathrm{GeV})<$ & 28 & 28 & $65.8 \%$ \\
$E_{\mu \mu}(\mathrm{GeV})<$ & 56 & 52 & $69 \%$ \\
\hline
\end{tabular}

Table 2: The kinematical cuts, each of them preserves separately $90 \%$ of muon pairs produced by muonic photons in the detector (columns 2 and 3), and the percentage of the trident background events within the cuts given for external bremstrahlung case (column 4). Here $M_{\mu \mu}$ is the invariant mass of a muon pair; $p_{t}^{\mu}$ and $p_{t}^{\mu \mu}$ are transversal momenta of a muon and a muon pair correspondingly, $\vartheta_{\mu \mu}$ is the angle between $\mu^{+}$and $\mu^{-}$, while $\varphi_{\mu \mu}$ is the angle between their transversal momenta; $E_{\mu}$ - energy of a muon $\left(\mu^{+}\right.$or $\left.\mu^{-}\right)$and $E_{\mu \mu}$ is energy of a muon pair. For internal bremsstrahlung the numerical results only slightly depend on neutrino mass within the discussed range, at least up to $10^{-10} \mathrm{eV}$.

and $\pi / \mathrm{K}$ lifetimes. So at the input every event has the coordinates of the $\pi / \mathrm{K}$ decay point and the vector of $\pi / \mathrm{K}$ momentum. The difference with the semi-analytical approach is that the radial and longitidinal spatial distributions of $\pi / \mathrm{K}$ in the decay tunnel, the angular distribution of $\pi / \mathrm{K}$ mesons and their correlations are automatically taken into account. Both positive and negative $\pi / \mathrm{K}$ mesons are used for muonic photon flux calculation in the neutrino Wide Band Beam (WBB), where positive mesons are focussed, while negative mesons are defocussed.

\begin{tabular}{|l|c|c|}
\hline Observable & Semi-analytical & Monte-Carlo \\
\hline$\overline{E_{\gamma}^{\text {int }}}$ & $16.8 \mathrm{GeV}$ & $17.9 \mathrm{GeV}$ \\
$E_{\gamma}^{\text {ext }}$ & $18.5 \mathrm{GeV}$ & $22.8 \mathrm{GeV}$ \\
$F_{\gamma}^{\text {znt }} / F_{\nu}$ & $10.4 \cdot 10^{-7}$ & $12.5 \cdot 10^{-7}$ \\
$F_{\gamma}^{\text {ext }} / F_{\nu}$ & $3.1 \cdot 10^{-7}$ & $2.3 \cdot 10^{-7}$ \\
$\sigma_{\gamma}^{\text {int }}$ & $35.6 \mathrm{pb}$ & $39.9 \mathrm{pb}$ \\
$\sigma_{\gamma}^{\text {ext }}$ & $41.5 \mathrm{pb}$ & $53.8 \mathrm{pb}$ \\
$N^{\text {int }}$ & $511 \mathrm{events}$ & 688 events \\
$N^{\text {ext }}$ & 177 events & 172 events \\
$N_{\text {total }}^{\text {trids }}$ & 140 events & 138 events \\
$N_{4 \text { GeV }}^{\text {tridents }}$ & 49 events & 47 events \\
\hline
\end{tabular}

Table 3: Results of semi-analytical and Monte-Carlo calculations of the following quantities: the average energies of muonic photons $\left(\overline{E_{\gamma}^{\text {int }}}, \overline{E_{\gamma}^{\text {ext }}}\right)$, the ratios of muonic photon flux to neutrino flux for external $F_{\gamma}^{\text {int }} / F_{\nu}$ and internal $F_{\gamma}^{e x t} / F_{\nu}$ bremsstrahlung, the average muonic gamma interaction cross-sections for gammas from internal $\sigma_{\gamma}^{i n t}$ and external $\sigma_{\gamma}^{e x t}$ bremsstrahlung, the numbers of events of muonic gamma conversions from internal $N^{i n t}$ and external $N^{\text {ext }}$ bremstrahlung and the numbers of trident events without $\left(N_{\text {total }}^{\text {tridents }}\right)$ and with $\left(N_{4 \mathrm{GeV}}^{\text {trident }}\right)$ the cut on muon momentum of $4 \mathrm{GeV}$. The fluxes, cross-sections and mean energies were calculated with the cut $E_{\gamma}>7 \mathrm{GeV}$ and $E_{\mu}>4 \mathrm{GeV}$.

The internal bremsstrahlung of muonic gammas in $\pi / \mathrm{K}$ decays is generated according to the formulas used in the section 2. The flux of the muonic photons is calculated in the fiducial volume of CHARM-II detector with square cross-section of $3.2 \times 3.2 \mathrm{~m}^{2}$ located at $882 \mathrm{~m}$ from the proton target.

The production of the muonic photons by external bremsstrahlung from muons, stopping in the iron shielding 
was calculated according to the formula (9). For this study the shielding was appriximated by 80 blocks of iron, each $3 \mathrm{~m}$ in length. The simplified tracking take into account the energy loss of $4.5 \mathrm{GeV}$ per block and multiple scattering of muons in the iron. The muons were tracked down to $10 \mathrm{GeV}$. Muonic photons were generated with the angular distribution (f.w.h.m. $0.12^{\circ}$ ) around the muon direction at each tracking step. The effect of the multiple scattering reduce the flux of muonic photons in the volume of the neutrino detector, because of the increase of the angular spread.

The conversion of muonic photons into muon pairs in the target of the neutrino detector was simulated according to formula (14) for the average CHARM II nuclei $(A=20, Z=10)$. The energy cut of $4 \mathrm{GeV}$ was applied on both muons.

The cross-section and rates of the trident background were calculated by Monte-Carlo program [28] with neutrino spectra predicted by GBEAM [8] program. Only coherent part of the cross-section was estimated for this study.

The results of these calculations in the form of the mean energies, cross-sections and rates are summarised in the table 3 for the neutrino mass $m_{\nu_{\mu}}=10^{-10} \mathrm{eV}$ and $\alpha_{\mu} / \alpha=10^{-5}$. The numerical results of this Monte-Carlo simulation are close to the semi-analytical calculations. The differences are due to different approximations and assumptions on the geometry, the spectra of mesons, the effects of multiple scattering and the angular distributions.

\section{Conclusions.}

Let us summarize the main results of this paper. If $\alpha_{\mu} \sim 10^{-5} \alpha$, as allowed by $(g-2)_{\mu}$ data, then in the high-energy neutrino experiment of the type of CHARM II one may observe $\sim 180$ narrow muon pairs produced by muonic photons in the case of external bremsstrahlung. In the case of internal bremsstrahlung there may observe about 70 events if the mass of muonic neutrino is $100 \mathrm{KeV}$, and $\sim 600$ events if the mass of muonic neutrino is very small, of order $10^{-10} \mathrm{eV}$. This should be compared with approximately 50 muon pairs from trident events. Thus, muonic photons could be discovered in such experiments if $\alpha_{\mu} \sim 10^{-5} \alpha$. Moreover, one may hope to measure the mass of muonic neutrino if this mass is small enough and muonic photon is discovered.

By selecting pairs with $p_{t}<70 \mathrm{MeV}$ one may reduce the number of background trident pairs by a factor of 50 , to a few events. One may hope to further suppress the trident background by selecting muon pairs with invariant mass less than $500 \mathrm{MeV}$, or with azimuth angle between muons larger than $140^{\circ}$, or with transversal momentum of muon and antimuon smaller than $200 \mathrm{MeV}$. Thus, if no events satisfying the cuts are observed, one can reach the sensitivity of $\alpha_{\mu} / \alpha<10^{-6}$.

\section{Acknowledgments}

The authors are grateful to K. Winter whose questions about the difference between the muon pairs from muonic photons and tridents initiated this work. L.O. is grateful to R.R. Kokoulin, S.R. Kelner, S.A. Maltsev, A.Yu. Morozov, L.I. Rozental, A.V. Smilga, M.J. Tannenbaum, V.R. Zoller for interesting discussions, and to RFBR for the grants 96-02-18010 and 96-15-96578. V.I. is grateful to E.E. Boos and A.E. Pukhov for useful discussions, and to the European association INTAS (contract 93-1180ext) and the Grant Center for Natural Sciences of State Committee for Higher Education in Russia (grant 95-0-6.4-38). A.R. is grateful to CHARM-II/CHORUS and CPPM colleagues for interesting discussions and support. 


\section{References}

[1] T.D. Lee and C.N. Yang. Phys.Rev. 98 (1955) 1501.

[2] L.B. Okun. Yadernaya Fizika 10 (1969) 359 (in Russian); Sov. Journal of Nucl.Phys. 10 (1969) 206.

[3] L.B. Okun, Phys.Lett. B382 (1996) 389-392;

[4] L.B. Okun. 'Leptons and Quarks'. North Holland. 1984. Chapter 19, p. 166.

[5] A.K. Ciftci, S. Sultansoi, and S. Türköz. Phys. Lett. B355 (1995) 494.

[6] S.I. Blinnikov, A.D. Dolgov, L.B. Okun, and M.B. Voloshin. Nucl. Phys. B458 (1996) 5264.

L.B. Okun, 'A remark on leptonic photons'. June 1972 (Unpublished manuscript in Russian, presented at the ITEP theoretical seminar).

[7] L.B. Okun. 'Leptons and Photons'. Preprint ITEP, hep-ph/9512436.

[8] C.Foos et al., GBEAM - neutrino beam simulation program, CHARM II Internal Note, Nov 1989.

[9] Review of Particle Properties. Phys. Rev. D54, No. 1 (1996), Part 1.

[10] E.E. Boos, M.N. Dubinin, V.A. Ilyin, A.E. Pukhov, and V.I. Savrin. 'CompHEP - specialised package for automatic calculation of elementary particle decays and collisions'. Preprints SNUTP 94-116, Seoul, 1994; INP MSU 94-36/358; hep-ph/9503280.

[11] V.A. Ilyin, D.N. Kovalenko and A.E. Pukhov, I.J.Mod.Phys.,C7 (1996) 761.

[12] S. Kawabata. Comp. Phys. Commun. 41 (1986) 127.

[13] A.C. Hearn and J.Fitch, 'REDUCE USER'S MANUAL', version 3.6. The Rand Publication, CP87 (Rev. 7/95), 1995.

[14] V.A.Ilyin, Note on the method of muon, neutrino and muon gamma spectra calculation with geometrical and angular acceptance (1997).

[15] H.A. Bethe and W. Heitler. Proc. Roy. Soc. London A146 (1934) 83.

[16] H.A. Bethe. Proc. Cambr. Phil. Soc. 30 (1934) 524.

[17] W. Heitler. 'The Quantum Theory of Radiation'. Thirds edition, Oxford, Clarendou Press, 1954, $§ 25$.

[18] A.I. Akhiezer and V.B. Berestetsky. 'Kvantovaya Electrodinamika'. Third edition, Nauka Publishers, Moscow $1969, \S 28$. (in Russian) 'Quantum Electrodynamics', §29, Interscience Publisher, New York, 1965.

[19] A.A. Petrukhin and V.V. Shestakov, Can. J. Phys. 46 (1968) §377.

[20] I.L. Rozental. Uspekhi Fiz. Nauk 94 (1968) 91 (in Russian); Sov. Phys. Uspekhi 11 (1968) 49.

[21] R.P. Kokoulin and A.A. Petrukhin. In: Proceedings of the International Conference on Cosmic Rays (Hobart, Australia, August 1971), vol. 4, p. 2436.

[22] S.R. Kelner, R.P. Kokoulin and A.A. Petrukhin. 'About cross-section for high-energy muon bremsstrahlung'. Preprint 024-95, Moscow State Engineering Physics Institute. Moscow 1995.

[23] Yu.M. Andreev, L.B. Bezrukov and E.V. Bugaev. Yadernaya Fizika 57 (1994) 2146 (in Russian); Phys. of Atomic Nuclei 57 (1994) 2066.

[24] M.J. Tannenbaum. CERN-PPE/91-134 (1991) (unpublished).

[25] See [9], p. 137.

[26] H. de Vries, C.W. de Jager, and C. de Vries. 'Nuclear Charge-density-distribution parameters from elastic electron scattering'. In: 'Atomic Data and Nuclear Data Tables', 36 (1987) 495.

[27] R.P. Kokoulin and A.A. Petrukhin. In: 'Kosmofizika', ed. V.V. Dmitrienko, Energoatomizdat, Moscow, 1987, p. 76 (in Russian).

[28] CHARM II Collaboration, A new measurement of the cross-section for trilepton production induced by muon neutrinos (in preparation).

[29] See [ $₫$, Chapter 16, p. 140. Corrected in the second Russian edition: 'Leptony i kvarki', Moskva, Nauka, 1990, p.158. 